\title{
Infecção por Kudoa Meglitsch, 1947 (Myxozoa: Multivalvulida) em musculatura esquelética de espada Trichiurus lepturus L. (Teleostei: Trichiuridae)
}

\author{
CAROLINE DEL GIUDICE DE ANDRADA*, ROGERIO TORTELLY** PATRÍCIA PEREIRA NOGUEIRA*, \\ CLAUDIA LEAL ANDRADE* e FRANCISCO CARLOS DE LIMA****
}

\section{Kudoa MEGLITSCH, 1947 (MYXOZOA: MULTIVALVULIDA) INFECTION IN SOMATIC MUSCLES OF CUTLESS FISH Trichiurus lepturus (TELEOSTEI: TRICHIURIDAE)}

The presence of Kudoa sp in somatic muscle of cutless fish (Trichiurus lepturus) captured at Itaipu beach, Niterói, Rio de Janeiro, is been reported. It was used as samples 47 specimes, measured and necropsied at the Laboratório de Inspeção e Tecnologia de Pescado da Faculdade de Veterinária daUniversidade Federal Fluminense. The tissues were processed for paraffin inclusion and stained by hematoxilina-eosin. From the total samples, nine of them $(19,15 \%)$ showed plasmodic type cists in muscular fibers without inflammatory reaction. It was four valves, the principal feature of the genus. The number of cysts varied from one to 15, with a average of 5,1. The length and width average of cysts were 82,57 and 28,27, respectively. The length average of positive fishes was $116 \mathrm{~cm}$. The smaller animal $(68,0)$ showed more cysts. It was not observed myoliquefaction even several hours after captured.

Key words: Kudoa, Trichiurus lepturus, Brazil.

\section{INTRODUÇÃO}

O gênero Kudoa compreende os parasitos mixosporídeos que apresentam quatro válvulas, sendo conhecidas, até a presente data, 46 espécies, todas parasitas de peixes.

É um gênero cosmopolita, encontrado em peixes teleósteos marinhos ${ }^{1-4}$ e estuarinos ${ }^{5}$, e mesmo em peixes cartilaginosos ${ }^{6}$. Algumas espécies de Kudoa são de especial importância devido ao impacto na qualidade dos produtos pesqueiros, representado pelo aparecimento de mioliquefação pós-mortem ${ }^{7-10}$, tornando-os inaceitáveis para o consumo humano. Esta lise muscular é resultado da ação de proteases ${ }^{11}$, produzidas e utilizadas pelos parasitos para amolecer o músculo do hospedeiro, facilitando, assim, o seu desenvolvimento ${ }^{12}$.

Embora a maioria das espécies de Kudoa tenha como sítio de infecção a fibra muscular esquelética, como é o caso de $K$. scianae em Stellifer minor ${ }^{1}, K$. shkae em Arius felis e $K$. leiostomi em Leiostomus xanthurus ${ }^{13}, K$. miniauricula em Sebastes paucispinis ${ }^{14}, K$.

\footnotetext{
* Médicas veterinárias.

** Serviço de Anatomia Patológica Veterinária. Prof. Jefferson Andrade dos Santos - Dept ${ }^{\circ}$ de Patologia - Fac. Veterinária/UFF.

*** Dept ${ }^{\circ}$ Tecnol. de Alimentos - Fac. Veterinária/UFF - Rua Vital Brazil Filho, 64/Niterói-RJ.
} 
paniformis em Merluccius productus ${ }^{15-17}, K$. ramsayi em Patagonotothen ramsayi ${ }^{18}$, outros tecidos podem ser afetados, como a musculatura lisa gástrica e intestinal, fígado, baço e tecido peripancreático de Morone americana ${ }^{5}$, serosa do intestino e cecos pilóricos de peixes do gênero Sillago ${ }^{19}$ e, em musculatura cardíaca de de Salmo salar $^{20}$.

A grande maioria das espécies de Kudoa possui hospedeiros específicos, no entanto, $K$. thyrsites parece ser a que apresenta menor especificidade, sendo registrada por, pelo menos, 23 espécies de várias famílias ${ }^{12}$.

A presente pesquisa reporta a presença de lesões microscópicas causadas por Kudoa sp. em fibras musculares somáticas de espada (Trichiurus lepturus L.), do litoral do Rio de Janeiro.

\section{MATERIAL E MÉTODOS}

Foram analisados 47 espécimes de peixe espada (T.lepturus) capturados na praia de Itaipu, Niterói, RJ. Os animais foram acondicionados em gelo e transportados para o Laboratório de Tecnologia e Inspeção de Pescado da Faculdade de Veterinária da Universidade Federal Fluminense, onde foram medidos, tendo os comprimentos variado de 68,0 a $142,0 \mathrm{~cm}$, com a média de $116,0 \mathrm{~cm}$, e necropsiados. No Serviço de Anatomia Patológica Veterinária Professor Jefferson Andrade dos Santos, os tecidos musculares esqueléticos foram processados para a técnica de inclusão em parafina e coloração pela hematoxilina-eosina. De cada exemplar foi retirado um fragmento da porção muscular da região lateral mediana, com $1,0 \times 0,50 \mathrm{~cm}$. $\mathrm{Na}$ análise microscópica, os cistos encontrados em cinco diferentes campos foram contados e medidos, utilizando-se aumento de 200X.

\section{RESULTADOS E DISCUSSÃO}

Nove $(19,2 \%)$ espécimes de T. lepturus apresentavam cistos parasitários nas fibras musculares somáticas (Figura 1A), do tipo plasmódio, com ausência de reação inflamatória. Os parasitos presentes nestes cistos possuiam quatro válvulas (Figura 1B), com morfologia característica do gênero Kudoa.

A maioria dos relatos aponta alterações de natureza inflamatória, freqüentemente associadas

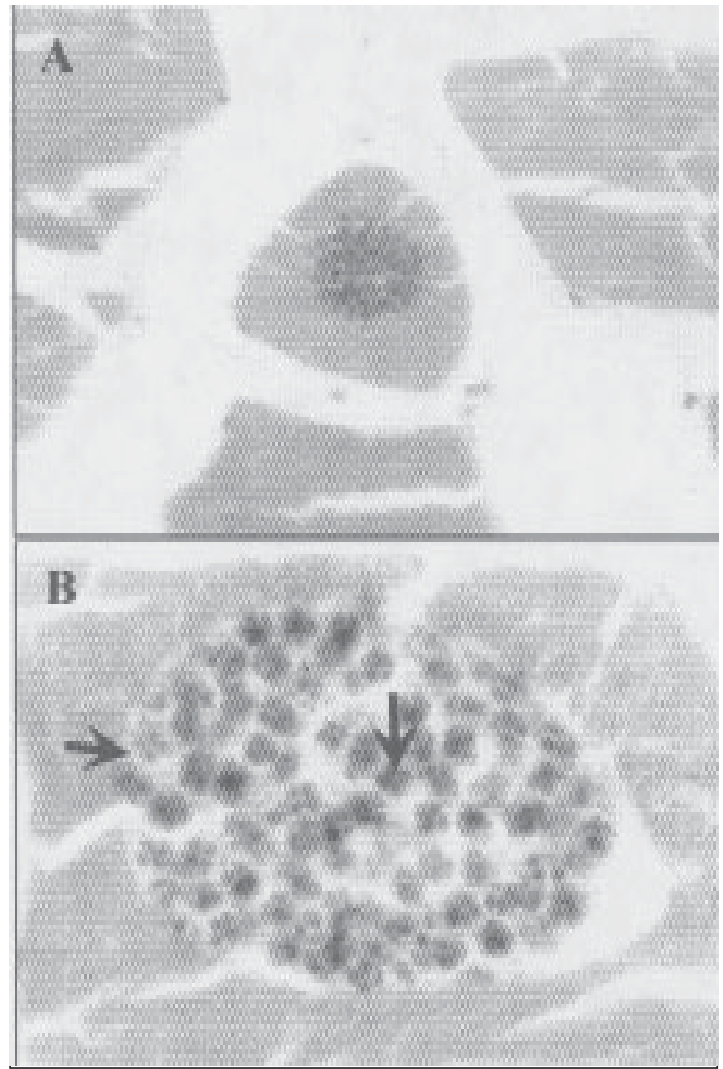

Figura 1A. Trichiurus lepturus. Corte histológico de musculatura mostrando plasmódio de Kudoa no centro de uma fibra muscular. Aumento: 100X. Figura $1 B$. Plasmódio repleto de parasitos, sendo visível as quatro válvulas (setas), características do gênero. Aumento: 400X.

à parasitose, com ampla variação da resposta tecidual. Em S. paucispinis foram registradas necrose e atrofia da musculatura esquelética ${ }^{21} \mathrm{e}$ encapsulamento fibrótico ${ }^{14}$; em $M$. productus foram verificadas a produção de infiltrado fagocitário, granulomas, formação capsular, pigmentação no interior de cistos degenerados, além de necrose de coagulação ${ }^{17}$; em Coryphaena hippurus foi constatada uma pronunciada resposta fibroblástica, com macrófagos epitelióides encapsulando os esporos do sarcolema, necrose de coagulação, degeneração hialina das miofibrilas, ruptura e hipocromasia das fibras colágenas; em M.americana foi observado infiltrado inflamatório mononuclear entre os feixes de fibras musculares esqueléticas ${ }^{5}$; em Salmo salar a reação do hospedeiro apresentava-se como uma inflamação crônica multifocal das fibras musculares. 
Resultados semelhantes aos do presente trabalho foram observados em Salmo trutta ${ }^{7}$ e em Hemiscyllium ocellatum ${ }^{6}$ que verificaram pouca ou nenhuma reação inflamatória associada à parasitose.

Nas infecções por Kudoa reportadas em $M$. productus $^{15,16}$, em S.paucispinis ${ }^{14}$, em Pomatoschistus minutus $^{9}$ e Cynoglossus senegalensis ${ }^{12}$, os cistos alcançavam tamanhos que permitiam a observação macroscópica. Em T. lepturus, as lesões eram sempre microscópicas.

A mioliquefação pós-mortem da musculatura esquelética $^{7-9}$ não foi observada em nenhum exemplar de T. lepturus.

A prevalência foi de $19 \%$. O número de cistos variou de um a quinze, com uma média de 5,1 cistos por peixe. As dimensões dos cistos foram de 40 a $168 \mu \mathrm{m}$ de comprimento, com média de $82,5 \mu \mathrm{m}$, e de 16 a $54 \mu \mathrm{m}$ de largura, com média de $28 \mu \mathrm{m}$. O animal de menor tamanho apresentou o maior número de cistos ${ }^{15}$.

\section{RESUMO}

A presente pesquisa reporta a presença de lesões causadas por Kudoa sp. nas fibras musculares somáticas de espada (Trichiurus lepturus L.), capturado na praia de Itaipu, Niterói, Rio de Janeiro. Utilizou-se, como amostragem, 47 exemplares, que foram medidos e necropsiados no Laboratório de Inspeção e Tecnologia de Pescado da Faculdade de Veterinária da Universidade Federal Fluminense. As amostras de tecidos foram processadas para a técnica de inclusão em parafina e coloração pela técnica de hematoxilina-eosina. Do total de espécimes amostrados, $9(19,15 \%)$ apresentavam, nas fibras musculares somáticas, cistos parasitários, do tipo plasmódio, com ausência de reação inflamatória. Os parasitos possuiam quatro válvulas, apresentando morfologia característica do gênero Kudoa. A variação no número de cistos foi de um a quinze, com média de 5,2. As médias dos comprimentos e das larguras dos cistos foram de $79,74 \mu \mathrm{m}$ e $31,22 \mu \mathrm{m}$, respectivamente. O menor animal $(68,0 \mathrm{~cm})$ apresentou o maior número de cistos. Não foi observada mioliquefação em T. lepturus.

\section{REFERÊNCIAS}

1.- OLIVA M, LUQUE J L, TERAN L, et al. Kudoa sciaenae (Myxozoa: Multivalvulidae) cysts distribution in the somatic muscles of Stellifer minor (Tschudi, 1844) (Pisces: Sciaenidae). Mem. Inst. Oswaldo Cruz, 1992; 87: 33-5.

2.- LANGDON J S, THORNE T, FLETCHER W J. Reservoir hosts and new clupeoid host records for the myoliquefactive myxosporean parasite Kudoa thyrsites (Gilchrist). J Fish Dis 1992; 15: 459-71.

3.- CASTRO R R, BURGOS R. Kudoa thyrsites (Myxozoa, Multivalvulida) causing 'milky condition' in the musculature of Paralichthys adspersus (Neopterygii, Pleuronectiformes, Paralichthydae) from Chile. Mem. Inst. Oswaldo Cruz 1996; 91: 163-4.

4.- PAMPOULIE C, MARQUES A, ROSECCHI E, et al. First record of Kudoa (Myxosporea) in Pomatoschistus microps in the Rhône Delta, France. J Fish Biol 1998; 53: 893-6.

5.- BUNTON T E, POYNTON S L. Kudoa sp. (Myxosporea, Multivalvulida) infection in juvenile white perch, Morone americana (Gmelin): histopathology and spore morphology. J Fish Dis 1991; 14: 589-94.

6.- HEUPEL M R, BENNETT M B. A myxosporean parasite (Myxosporea: Multivalvulida) in the skeletal muscle of epaulette sharks, Hemiscyllium ocellatum (Bonaterre), from the Great Barrier Reef. J Fish Dis 1996; 19: 189-91.

7.- HOLLIMAN A. Sea trout, Salmo trutta, a new host for the myxosperean Kudoa thyrsites (Gilchrist)? Vet Rec 1994; 14: 524-5.

8.- DAVIES A J, ANDREWS T, UPTON N P D, et al. Kudoa as a contributary cause of muscle necrosis in gobies. Bull Eur Ass Fish Pathol 1998; 18: 62-6.

9.- PAMPOULIE C, MARQUES A, ROSECCHI E, et al. First observation of Kudoa sp. okn a migratory Mediterranean goby, Pomatoschistus minutus (Pallas, 1770) in the Rhône Delta, France. J Eukar Microbiol 1999; 21-5.

10.- DAWSON-COATES J A, CHASE J C, FUNK V, et al. The relationship between flesh quality and numbers of Kudoa thyrsites plasmodia and spores in armes Atlantic salmon, Salmo salar L. J Fish Dis 2003; 26: 451-9.

11.- KONAGAYA S. [Jellification and protease activity of yellowfin sole muscle in association with a myxosporidian parasite]. Bull Jap Soc Sci Fish1980; 46: 1019-26.

12.- MORAN J D W, WHITAKER D J, KENT M L. A review of the myxosporean genus Kudoa Meglitsch, 1947 , and its impact on the international aquaculture industry and commercial fisheries. Aquaculture, 1999; 172: 163-96.

13.- DYKOVÁ I, LOM J, OVERSTREET $\mathrm{R} M$. Myxosporean parasites of the genus Kudoa Meglitsch, 1947 from some Gulf of Mexico fishes: Description of two new species and notes on their ultrastructure. Europ J Protist 1994; 30: 316-23.

14.- WHITAKER D J, KENT M L, SAKANARI J A. Kudoa miniauriculata n. sp. (Myxozoa, Myxosporea) from the musculature of bocaccio (Sebastes paucispinis) from California. J Parasitol 1996; 82: 312-5.

15.- KABATA Z, WHITAKER D J. Distribution of two species of Kudoa (Myxozoa: Multivalvulida) in the offshore population of the Pacific hake, Merluccius 
productus (Ayres, 1855) Can J Zool 1986; 64: 210310.

16.- STEHR C, WHITAKER D J. Host-parasite interaction of the myxosporeans Kudoa paniformis Kabata \& Whitaker, 1981 and Kudoa thyrsitis (Gilchrist, 1924) in the muscle of Pacific whiting, Merluccius productus (Ayres): an ultrastructural study. J Fish Dis 1986; 9: 505-17.

17.- MORADO J F, SPARKS A. Observations on the hostparasite relations of the Pacific whiting, Merluccius productus (Ayres), and two myxosporean parasites, Kudoa thyrsites (Gilchrist, 1924) and K. paniformis Kabata \& Whitaker, 1981. J Fish Dis 1986; 9: 445-55.

18.- CHAGANTI K, PAUL B, KEN M. Two new species of myxozoan parasites (Myxosporea, Multivalvulida,
Bivalvulida) from fishes of the Falkland Islands. Acta Parasitol 2000; 45: 285-8.

19.- HALLETT S L, O`DONOGHUE P J, LESTER R J G. Infections by Kudoa ciliatae (Mixozoa: Myxosporea) in Indo-Pacific whiting Sillago spp. Dis Aquat Org 1997; 30: 11-6.

20.- MORAN J D W, MARGOLIS L, WEBSTER J M, et al. Development of Kudoa thyrsites (Myxozoa: Myxosporea) in netpen-reared atlantic salmon determined by light microscopy and a polymerase chain reaction test. Dis Aquat Org 1999; 37: 185-93.

21.- HECKMANN R A, JENSEN L A. The histopathology and prevalence of henneguya sebasta and Kudoa clupeidae in the rockfish, Sebastes paucispinis of southern california. J Wildl Dis 1978; 14: 259-62. 